OPEN ACCESS

Edited by:

Pedro Miguel Duarte Moreno, University of Porto, Portugal

Reviewed by:

Sofia Duque Santos,

University of Porto, Portugal

Ester Kwon,

University of California, San Diego,

United States

*Correspondence:

Wilfred A. Jefferies

wilf@msl.ubc.ca

tThese authors have contributed equally to this work and share first authorship

Specialty section:

This article was submitted to Molecular Diagnostics and

Therapeutics,

a section of the journal

Frontiers in Molecular Biosciences

Received: 28 September 2020 Accepted: 16 February 2021

Published: 26 March 2021

Citation:

Eyford BA, Singh CSB, Abraham T, Munro L, Choi KB, Hill T,

Hildebrandt R, Welch I, Vitalis TZ, Gabathuler R, Gordon JA, Adomat H,

Guns EST, LU C-J, Pfeifer CG,

Tian MM and Jefferies WA (2021) A Nanomule Peptide Carrier Delivers siRNA Across the Intact Blood-Brain Barrier to Attenuate Ischemic Stroke.

Front. Mol. Biosci. 8:611367.

doi: 10.3389/fmolb.2021.611367

\section{A Nanomule Peptide Carrier Delivers siRNA Across the Intact Blood-Brain Barrier to Attenuate Ischemic Stroke}

\author{
Brett A. Eyford ${ }^{1,2,3,4 t}$, Chaahat S. B. Singh ${ }^{1,2,3,4,5 t}$, Thomas Abraham ${ }^{6 \dagger}$, Lonna Munro ${ }^{1,3}$, \\ Kyung Bok Choi ${ }^{1,3}$, Tracy Hill ${ }^{7}$, Rhonda Hildebrandt ${ }^{7}$, Ian Welch ${ }^{7}$, Timothy Z. Vitalis ${ }^{9}$, \\ Reinhard Gabathuler ${ }^{9,10}$, Jacob A. Gordon 2,11, Hans Adomat ${ }^{2,11}$, Emma S.T. Guns, 21 , \\ Chieh-Ju Lu ${ }^{1,2,3,4}$, Cheryl G. Pfeifer ${ }^{1,2,3,4}$, Mei Mei Tian $^{9}$ and Wilfred A. Jefferies ${ }^{1,2,3,4,5,11,12,13 *}$ \\ ${ }^{1}$ Michael Smith Laboratories, University of British Columbia, Vancouver, BC, Canada, ${ }^{2}$ The Vancouver Prostate Centre, \\ Vancouver General Hospital, Vancouver, BC, Canada, ${ }^{3}$ Centre for Blood Research, University of British Columbia, Vancouver, \\ BC, Canada, ${ }^{4}$ The Djavad Mowafaghian Centre for Brain Health, University of British Columbia, Vancouver, BC, Canada, \\ ${ }^{5}$ Department of Medical Genetics, University of British Columbia, Vancouver, BC, Canada, ${ }^{6}$ Department of Neural and Behavioral \\ Sciences and Microscopy Imaging Core Lab, Pennsylvania State College of Medicine, Hershey, PA, United States, ${ }^{7}$ Centre for \\ Comparative Medicine, University of British Columbia, Vancouver, BC, Canada, ${ }^{8}$ Department of Chemistry, University of British \\ Columbia, Vancouver, BC, Canada, ${ }^{9}$ Bioasis Technologies Inc., Guilford, CT, United States, ${ }^{10}$ King's College London, London, \\ United Kingdom, ${ }^{11}$ Department of Urologic Sciences, University of British Columbia, Vancouver, BC, Canada, ${ }^{12}$ Department of \\ Microbiology and Immunology, University of British Columbia, Vancouver, BC, Canada, ${ }^{13}$ Department of Zoology, University of \\ British Columbia, Vancouver, BC, Canada
}

The blood-brain barrier (BBB) hinders the distribution of therapeutics intended for treatment of neuroinflammation (NI) of the central nervous system. A twelve-amino acid peptide that transcytoses the BBB, termed MTfp, was chemically conjugated to siRNA to create a novel peptide-oligonucleotide conjugate (POC), directed to downregulate NOX4, a gene thought responsible for oxidative stress in ischemic stroke. The MTfp-NOX4 POC has the ability to cross the intact BBB and knockdown NOX4 expression in the brain. Following induction of ischemic stroke, animals pretreated with the POC exhibited significantly smaller infarcts; accompanied by increased protection against neurological deterioration and improved recovery. The data demonstrates that the MTfp can act as a nanomule to facilitate BBB transcytosis of siRNAs; where the NOX-4 specific siRNA moiety can elicit effective therapeutic knockdown of a gene responsible for oxidative stress in the central nervous system. This study is the first to conclusively demonstrate both siRNAcarrier delivery and therapeutic efficacy in any CNS disease model where the BBB remains intact and thus offers new avenues for potential treatments of oxidative stress underlying neuroinflammation in a variety of neuropathologies that are currently refractory to existing therapies.

Keywords: stroke, peptide-oligonucleotide conjugate, MTfp, blood-brain barrier, NOX4, siRNA

\section{INTRODUCTION}

A common denominator in neurodegenerative disorders such as stroke, traumatic brain injury, Parkinson's disease, Alzheimer's disease, Huntington's disease, multiple sclerosis, and amyotrophic lateral sclerosis, is the induction of oxidative stress triggering neuroinflammation (Guo et al., 2013; Kowal et al., 2013; Murray et al., 2013; Bennett et al., 2014; Rubiano et al., 2015; Whiteford et al., 2015; Chiurchiù et al., 2016; Global Burden of Disease Cancer Collabration et al., 2017; Ma et al., 
2017). Stroke is one of the leading causes of death in North America (Xu et al., 2018; Statistics Canada, 2018; Rios, 2019). It is caused by the impairment of cerebral blood flow (ischemic stroke) or the rupture of blood vessels in the brain (haemorrhagic stroke). The interruption of oxygenation and metabolites results in neuronal death. Ischemic stroke results in acute and chronic inflammation, as a result of activation and polarization towards pro-inflammatory (M1) microglia and subsequent activation of the NADPH oxidase (NOX) enzyme. The NOX enzymes are the primary source of reactive oxygen species (ROS) and are expressed by microglia after ischemic stroke (Li et al., 2009; Cooney et al., 2013). During ischemic stroke NOX2, NOX3, and NOX4 isotypes modulates their expression depending on cell type and time post-injury. NOX4 has been in neurons, astrocytes, and microglia, while NOX3 has been identified only in neurons, and NOX2 is expressed in microglia and neurons. As a result of these findings NOX4, is thought to be responsible for the majority of oxidative stress observed in acute traumatic brain injury (Cooney et al., 2013). Animals deficient in NOX4 are strongly protected from ischemic stroke (Kleinschnitz et al., 2010; Radermacher et al., 2013). A growing list of modalities are enabling pretreatment of acute ischemic stroke patients with a history of stroke or patients at risk for a stroke in attempt to reduce the severity of stroke when it occurs (Schmülling et al., 2003; Mackman, 2008; Fuentes et al., 2009; Milionis et al., 2009; Aoki and Uchino, 2011; Yoo, 2016; Qin et al., 2017; Tsivgoulis et al., 2018). Thus, approaches that limit stroke-related damage are emerging as important therapeutic tools.

The utility of short antisense/interfering RNA (siRNA) therapeutic approaches for central nervous system (CNS) diseases, such as stroke has been limited by their biodistribution in vivo. to the site of tissue damage alone. On their own, siRNA preferentially localizes to the kidney and liver, and their exclusion from the brain continues to hamper their potential and this represents a significant technical hurdle (Braasch et al., 2004). However, if brain delivery was possible, we hypothesized that reducing the expression of genes in the brain that potentiate the pathophysiology of stroke could be neuroprotective. NADPH oxidase type 4 (NOX4; Uniprot Q9JHI8) was chosen because this protein is known to be upregulated during acute ischemic stroke, it is a major source of oxidative stress leading to neuronal apoptosis (Radermacher et al., 2013; Qin et al., 2017; Yao et al., 2017).

We have previously shown that melanotransferrin (MTf; Uniprot P08582), a mammalian iron-transport protein (Food et al., 1994; Rothenberger et al., 1996; Demeule et al., 2002; Moroo et al., 2003; Yang et al., 2004), can deliver anti-cancer drugs that reduce the in situ growth of brain tumours (Karkan et al., 2008). It has been shown that a 12 amino acid peptide derived from MTf (MTfp) can also act as a nanomule (i.e., a tiny conveyor or ferry for drugs) to deliver a protein-based analgesic to the brain (Thom et al., 2019). Here we report the creation of a fully functional peptide-oligonucleotide conjugate (POC) composed of MTfp and NOX4-targeted siRNA. Our data support the utility of MTfp-targeted delivery of siRNA to the brain as an efficacious pretreatment approach to reduce pathologies of the brain and the CNS.

\section{METHODS AND MATERIALS}

\section{Animal Experiments}

All protocols and procedures involving the care and use of animals in these studies were reviewed and approved by the Animal Care Committees of Ottawa National Research Council of Canada or the University of British Columbia; both of which are governed by the Canadian Council of Animal Care. The mice were kept on a $12 \mathrm{~h}$ light-dark cycle and had food ad libitum.

For the delivery of siRNA to the brain experiments, 6-8 weekold female BALB/c mice (Jackson Laboratory; \#000651) (16-20 g in weight) were used. For the induction of stroke experiments, 10 week-old male C57Bl/6 mice (Jackson Laboratory; \#000664) were used.

\section{Synthesis of MTfp Conjugates}

MTfp, NOX4 siRNA (5'-GACCUGACUUUGUGAACAU-3'), scrambled NOX4 siRNA (scramRNA, 5'-GUAAAUUCUCGC GACUAGU- $3^{\prime}$ ) and conjugates thereof were synthesized by Biosynthesis Inc. (Lewisville, United States). MTfp and RNA were produced independently and then chemically conjugated using the crosslinker succinimidyl-4-(N-maleimidomethyl) cyclohexane-1-carboxylate (Figure 1). High performance liquid chromatography and mass spectrometry indicated a purity of greater than $94 \%$. The final products were lyophilized and stored at $-80^{\circ} \mathrm{C}$.

\section{D Fluorescence Microscopy Imaging}

To determine the extent of delivery of RNA to the brain, 6-8 week-old female $\mathrm{BALB} / \mathrm{c}$ mice (16-20 $\mathrm{g}$ in weight) received third $\mathrm{IV}$ injections at $1 \mathrm{~h}$ intervals with $0.1 \mathrm{ml} /$ mouse PBS, siRNA ${ }_{\mathrm{AF} 680}(30 \mathrm{mg} / \mathrm{kg})$ or MTfp-siRNA $\mathrm{AF}_{680}(30 \mathrm{mg} / \mathrm{kg})$. Two hours post injection, mice received a second IV injection of tomato lectin conjugated to FITC $(100 \mu \mathrm{g} /$ mouse, $0.1 \mathrm{ml})$. The bloodstream was flushed by intracardiac perfusion for $10 \mathrm{~min}$ at a flow rate of $1 \mathrm{ml} / \mathrm{min}$ with heparinized saline $(0.9 \% \mathrm{NaCl}, 100 \mathrm{U} /$ $\mathrm{ml}$ heparin), until the extremities appeared white, as we have done previously (Ujiie et al., 2003; Karkan et al., 2008).

The mice were then sacrificed and the brains were removed and fixed with $4 \%$ paraformaldehyde overnight and then transferred into PBS $+0.01 \%$ sodium azide and stored at $4^{\circ} \mathrm{C}$. The brains were embedded in $4 \%$ agarose, fixed onto the microtome stage and sectioned $(20 \mu \mathrm{m})$ at $4^{\circ} \mathrm{C}$. Sections of the cerebral cortex were stained with DAPI and then mounted on microscopic slides. Glass coverslips were mounted on the sections using Prolong Gold antifade reagent.

The imaging experiments, which quantitatively assessed the ability of MTfp to direct the delivery of a therapeutic compound (NOX4 siRNA) to the brain as well as various localization issues, were performed using the Leica SP8 X system (Leica, Germany). 3D confocal images were acquired with a Leica AOBS SP8 laser scanning confocal microscope (Leica, Heidelberg, Germany) using a highresolution Leica $63 \mathrm{X} / 1.4$ or $40 \mathrm{X} / 1.3$ Plan-Apochromat oil immersion objective lens. Excitations were performed using either diode or tunable white light laser sources. All images and spectral data (except DAPI) were generated using the highly sensitive $\mathrm{HyD}$ detectors (with gated option) in de-scanned mode. The 


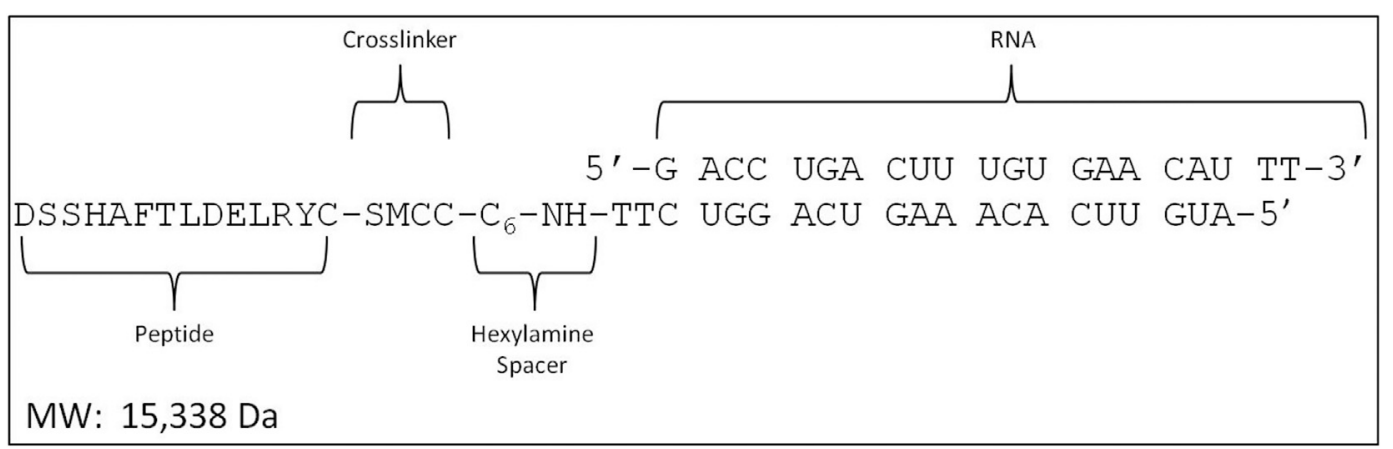

FIGURE 1 | Design of the MTfp-RNA conjugates. SMCC, succinimidyl 4-(N-maleimidomethyl) cyclohexane-1-carboxylate.

backscattered emission signals from the sample were delivered through the tunable filter (AOBS), the detection pinhole, spectral dispersion prism, and finally to the $\mathrm{PMT} / \mathrm{HyD}$ detectors. For $3 \mathrm{D}$ image data set acquisition, the excitation beam was first focused at the maximum signal intensity focal position within the brain tissue sample and the appropriate $\mathrm{HyD}$ gain levels were then selected to obtain the pixel intensities within range of 0-255 (8-bit images) using a color gradient function. The beginning and end of the $3 \mathrm{D}$ stack were set based on the signal level degradation. Series of 2D images for a selected 3D stack volume were then acquired with $1024 \times 1024$ pixels. The 3D stack images with optical section thickness ( $z$-axis) of approximately $0.3 \mu \mathrm{m}$ were captured from tissue volumes.

For each tissue volume reported here, $\mathrm{z}$-section images were compiled and finally the $3 \mathrm{D}$ image restoration was performed using VOLOCITY software (Perkin Elmer, United Kingdom). The volume estimation was performed on the $3 \mathrm{D}$ image data sets recorded from four or more either cortical or hypothalamus areas of brain tissue samples. Algorithms were developed to automate the quantification of the test articles, blood capillaries and to accurately quantify test articles localized in blood capillaries vs. brain parenchyma. In these procedures, a noise removal filter (either Gaussian or kernel size of $3 \times 3$ ) was used to remove the noise associated with the images. To define the boundary between the objects (for instance, blood capillaries) and the background, the lower threshold level in the histogram was set to exclude all possible background voxel values. The sum of all the voxels above this threshold level is determined to be the volume. Fields from each experimental group were pooled. ANOVA was used to assess the volume fractions in the brain parenchyma (Prism 6, Graphpad, La Jolla, United States). The data were log transformed to meet the assumption of homogeneity of variance. Post hoc analysis used a Dunnett's Test for multiple comparisons. The level of significance was $p<0.05$ for the ANOVA and the $p$-values were corrected for multiple comparisons in the post hoc analysis.

\section{RNA Isolation and qPCR}

Total RNA was isolated using $\mathrm{TRIzol}^{\circledR}$ reagent (Invitrogen, Burlington, Canada) from frozen brains of mice that had been treated with PBS, NOX4 siRNA, MTfp-NOX4 siRNA or MTfpscramRNA. RNA was reverse transcribed to cDNA with a Script
cDNA SuperMix agent (QuantaBio, Gaithersburg, United States) using random hexamers and oligo dT. SYBR ${ }^{\circledR}$ Green PCR Master Mix and RT-PCR Reagents kit (Applied Biosystems, Foster city, United States) were used to do quantitative real time PCR (qPCR) on an ABI 7500 FAST REAL TIME PCR System (Applied Biosystems, Foster city Unites States). Nox4 primers (5'-CTT GACTTGATGGAGGCAGTAG- $3^{\prime}$ and $5^{\prime}$-GCCTTTATTGTG CGGAGAGA- $3^{\prime}$ ) and GAPDH primers (5'-AACTTTGGCATT GTGGAAGG- $3^{\prime}$ and $5^{\prime}$-ACACATTGGGGGTAGGAACA- $3^{\prime}$ ) were used. The master reaction mixture consisted of $1 \mathrm{X}$ SYBR ${ }^{\circledR}$ Green PRC buffer, $3 \mathrm{mM} \mathrm{MgCl}_{2}, 1 \mathrm{mM}$ dNTP, $0.625 \mathrm{U}$ Taq polymerase, $0.25 \mathrm{U}$ amperase UNG, $10 \mathrm{ng} \mathrm{cDNA}$, and $300 \mathrm{nM}$ primers (sense and antisense). The $\mathrm{qPCR}$ was carried out at $50^{\circ} \mathrm{C}$ for $2 \mathrm{~min}, 95^{\circ} \mathrm{C}$ for $10 \mathrm{~min}$, followed by 40 cycles at $95^{\circ} \mathrm{C}$ for $15 \mathrm{~s}$, and $60^{\circ} \mathrm{C}$ for $1 \mathrm{~min}$. The qPCR efficiency was determined for each gene with the slope of a linear regression model, and all samples displayed efficiencies between 84 and 96\%. Negative controls containing no target RNA were included with each of the qPCR runs. The $\mathrm{qPCR}$ values were compared using the method described by Huggett et al. (Huggett et al., 2005). Data are expressed as mean \pm standard error of mean. In multiple group comparisons, data were analyzed by one-way ANOVA with Tukey post-hoc test. $p$-values less than 0.05 were considered significant.

\section{Stroke Induction by Transient Middle Cerebral Artery Occlusion}

Work with the mouse stroke model was performed at the National Research Council (Ottawa, Canada) and at the University of British Columbia (Vancouver, Canada), by different operators. Male $\mathrm{C} 57 \mathrm{Bl} / 6$ mice ( 10 weeks old) were injected by IV three times, at $1 \mathrm{~h}$ intervals, with PBS, NOX4 siRNA, MTfp-siRNA or MTfpscram RNA $(30 \mathrm{mg} / \mathrm{kg})$. One hour after the last injection, an ischemic stroke was induced on the left hemisphere by MCAO. The external and internal carotid arteries (ECA and ICA, respectively), were dissected and isolated and the common carotid artery (CCA) ligated. A nylon thread coated with dental silicon (Cat. \# 60231012, Doccol Corp) was inserted into a small hole in the ECA towards the bifurcation of the CCA. The thread was gently introduced into the ICA, passed into the Circle of Willis thus occluding the opening of the middle cerebral artery. Body 
temperature was not allowed to drop below $36^{\circ} \mathrm{C}$ and anesthesia was maintained throughout the surgery occlusion. The filament was removed after $1 \mathrm{~h}$, and the ligature on the CCA was removed allowing reperfusion of the affected brain area. Mice were sacrificed at $24 \mathrm{~h}$ post reperfusion. Sham surgeries (arteries isolated but not occluded) on animals that received all three injections of PBS were also performed as controls and animals were under anesthesia for as long as the stroke-induced animals.

\section{Stroke Analysis}

An individual, who was blinded to the treatment each mouse had received, assessed post stroke behaviour. Mice were assessed at 0.5 and $24 \mathrm{~h}$ after recovery from anesthesia using a method described by Jiang et al (Jiang et al., 2005). A score of zero is normal; one is mild turning behaviour with or without inconsistent curling when picked up by tail and $<50 \%$ attempts to curl to the contralateral side; two is mild consistent curling with $>50 \%$ attempts to curl to contralateral side; three is strong and immediate consistent curling, mouse holds curled position for more than 1-2 s, the nose of the mouse almost reaches tail; four is severe curling progressing into barrelling, loss of walking or righting reflex; five is comatose or moribund. According to this method, the neuroscore at $0.5 \mathrm{~h}$ is a measure of surgical success and identifies animals that had received abnormally severe or mild damage during the procedure. Mice were excluded from the final calculation for having a neurological scores below 1 (no effective stroke) or above 2.5 (surgical trauma).

To determine infarct size, mice were sacrificed after the second behavioural assessment ( $24 \mathrm{~h}$ post-op). Mice were terminally sedated with $0.2 \mathrm{ml}$ of ketamine $(200 \mathrm{mg} / \mathrm{kg})$ and Rhompun $(20 \mathrm{mg} / \mathrm{kg})$ and perfused transcardially at $1 \mathrm{ml} / \mathrm{min}$ for $10 \mathrm{~min}$ with heparinized saline $(0.9 \% \mathrm{NaCl}, 100 \mathrm{U} / \mathrm{ml}$ heparin). Brains were removed, sectioned and stained with $2 \%$ TTC in PBS to visualize the infarcts. Images of the brain slides were obtained after $15-20 \mathrm{~min}$ staining at room temperature. The brain slices were then cut in half to separate the ischemic hemisphere and the contralateral hemisphere. Tissue was rinsed with physiological saline and then exposed to a mixture of ethanol/dimethyl sulfoxide (1:1) for $24 \mathrm{~h}$ in the dark to solubilise the coloured formazan product. The absorbance at $485 \mathrm{~nm}$ was measured. Percentage loss in brain TTC staining in the ischemic side of the brain was compared with the contralateral side of the brain of the same animal using the following equation.

$$
\% \text { loss }=100 \times\left(1-\frac{\text { absorbance }: \text { ischemic hemisphere }}{\text { absorbance }: \text { contralateral hemisphere }}\right)
$$

Data are expressed as mean \pm standard error of mean. In multiple group comparisons, data were analyzed by one-way ANOVA with Tukey post-hoc test. $p$-values less than 0.05 were considered significant.

\section{RESULTS}

\section{MTfp-NOX4 siRNA Reduces Stroke Damage}

We have described the identification and transport characteristics of the 12 amino acid MTfp elsewhere (Thom et al., 2019). To

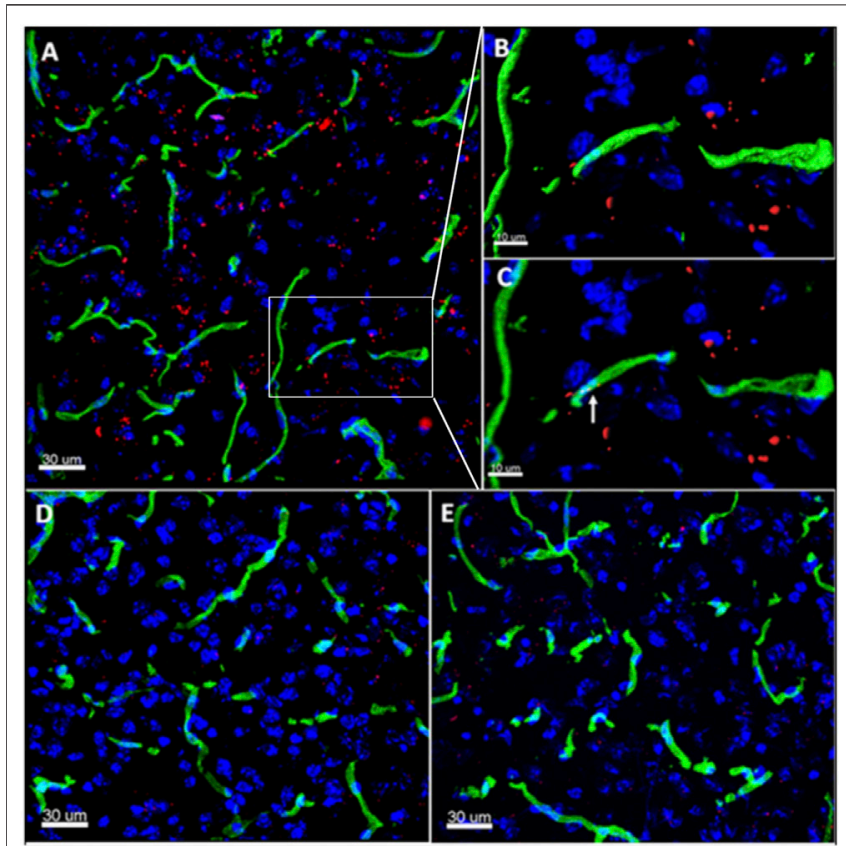

$\mathbf{F}$

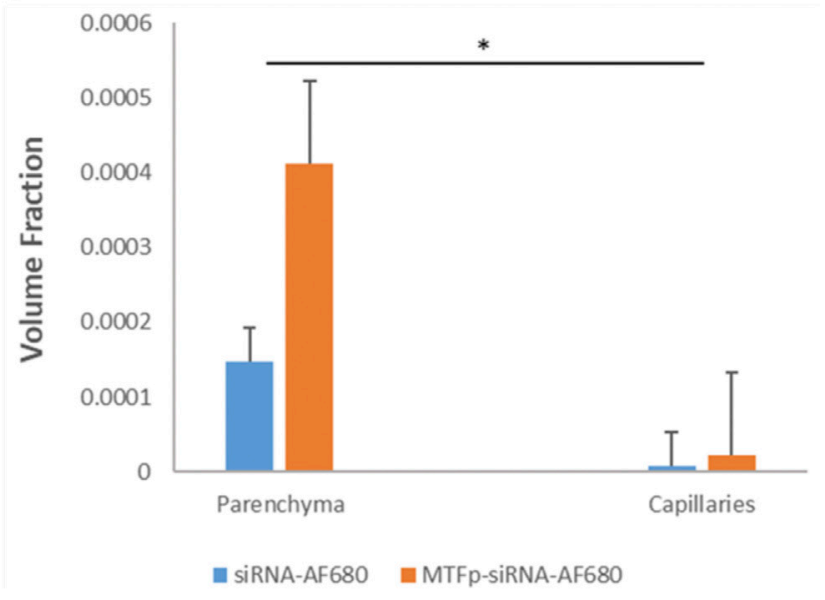

FIGURE 2 | Conjugation to MTfp enables siRNA to cross an intact BBB and into the brain parenchyma. Representative 3D confocal images showing localization of MTfp-siRNA in the brain of mice with intact BBB. Cell nuclei are blue (DAPI) and capillaries are green (Tomato lectin-FITC). (A) AF680

fluorescence (red) in the cerebral cortex sections from a mouse treated with MTfp-siRNA $A_{A F 680}$; (B) Shows the enlarged area that has been surfacerendered to indicate the surface of the capillaries where FITC labelled capillaries (green) and MTfp-siRNA $A_{A F 680}$ (red); (C) Shows the enlarged area

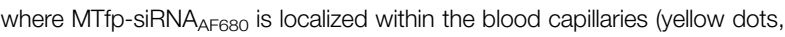
see arrow); (D) AF680 fluorescence (red) in the mouse cerebral cortex treated with PBS (i.e., background fluorescence); (E) Control AF680 fluorescence (red) in the mouse cortex treated with siRNA $A_{A F 680}$ (F) Distribution of MTfpsiRNA in the cortex of wild type mice with intact BBB. Values indicate total AF680 fluorescence normalized to total tissue volume (VTA in

Supplementary Table S1) and then normalized to the total AF680

fluorescence seen in PBS (background). Data are represented as means \pm SD $\left(n=3\right.$, eight fields of view per animal). ${ }^{*} p$-value $<0.05$.

assess whether MTfp could deliver RNA cargo to the CNS, MTfp was conjugated to AF680-conjugated NOX4-specific siRNA (Figure 1) and injected IV into mice. The brains were then 


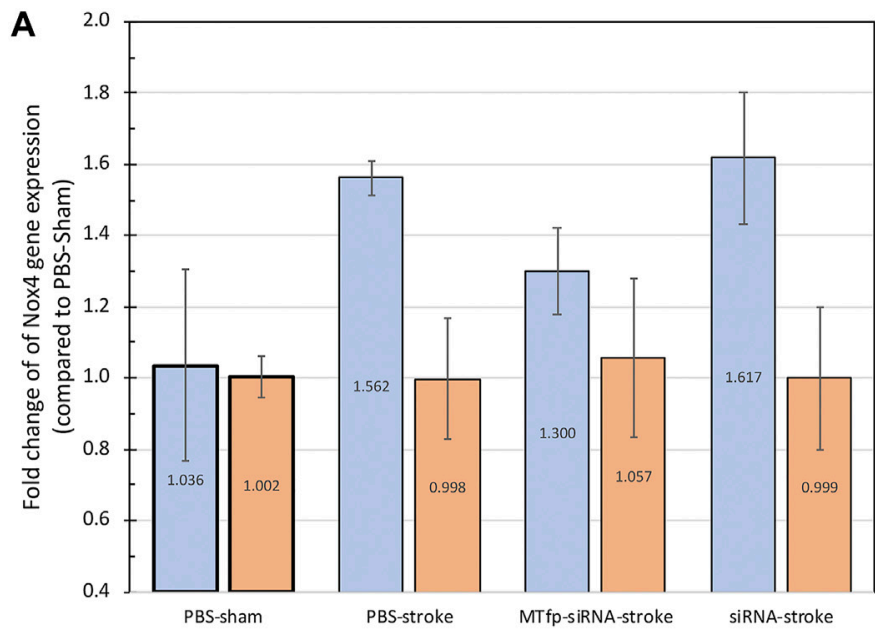

口Left Ischemic

$\square$ Right Contralateral

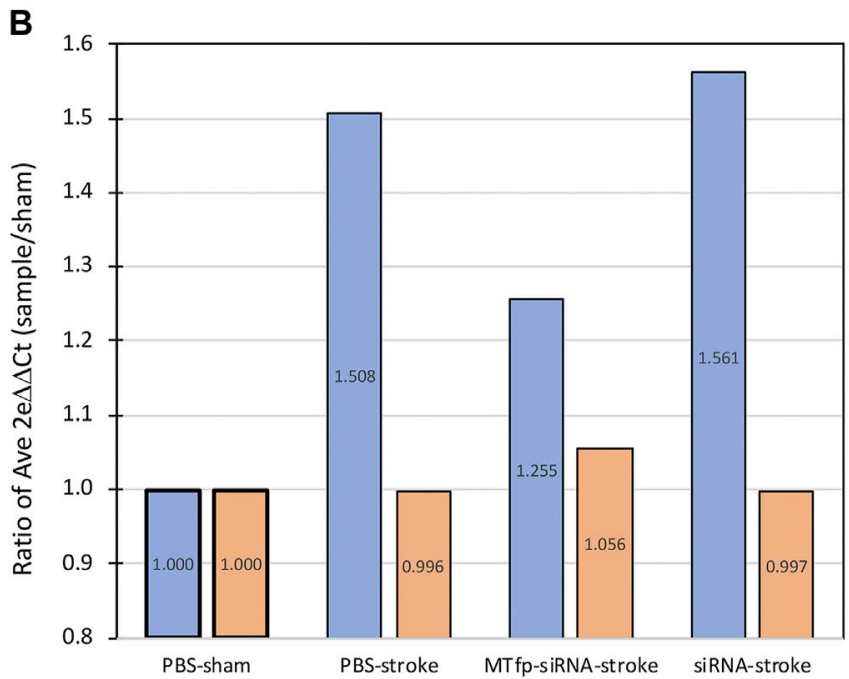

- Left Ischemic

$\square$ Right Contralateral

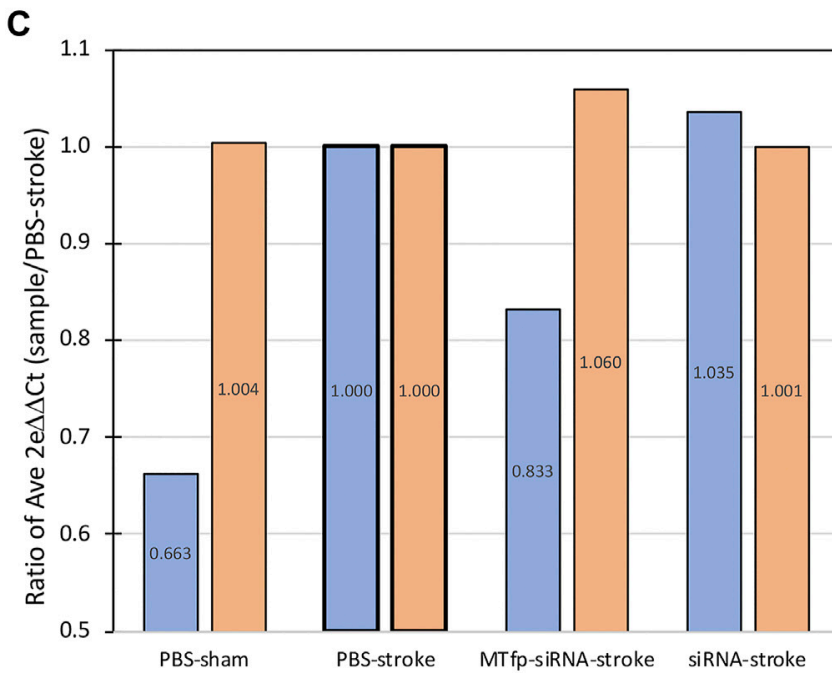

$\square$ Left Ischemic

$\square$ Right Contralateral

FIGURE 3 | NOX4 mRNA expression in the brain after siRNA treatment. The graphs show the fold change of Nox4 gene expression in the mouse brain hemispheres measured by qPCR following treatment and $24 \mathrm{~h}$ post-stroke, showing both the expression in left ischemic side and the right contralateral side. (A) Values are normalized to $\beta$-actin gene expression and individual samples are compared to the corresponding average PBS-sham condition (ischemic or contralateral). Data are shown as mean \pm SEM. ( $n=2-4$ mice per group). (B) Ratio of average fold change of each group compared to PBS-sham. (C) Ratio of average fold change of each group compared to PBS-stroke. "Left ischemic" in blue, refers to the measurments in the left stroke-induced hemisphere and "Right contralateral" in orange, refers to the measurement in the right non-stroke hemisphere. Raw data and calculations are shown in Supplementary Table S3. 


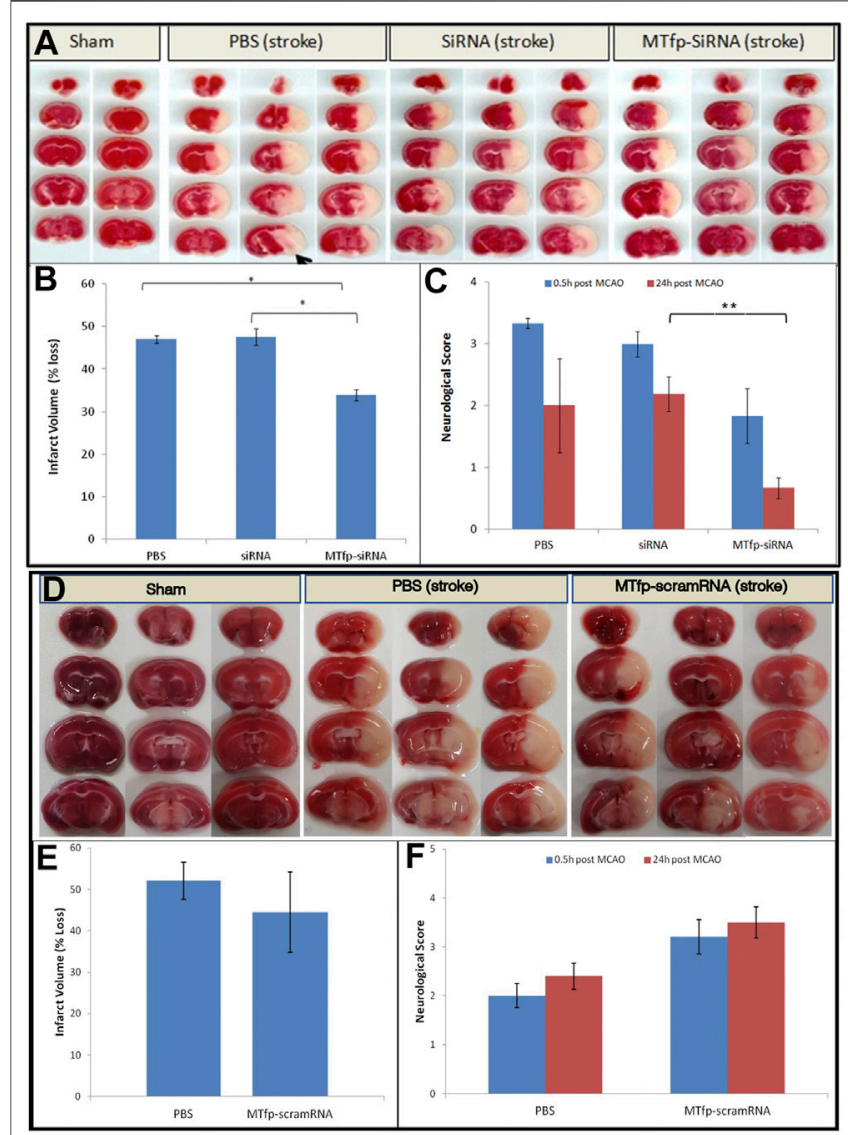

FIGURE 4 | Top panel. (A-C): Treatment with MTfp-siRNA confers neuroprotection and reduces damage after ischemic stroke. (A) TTC-stained brain sections of mice receiving various IV injections, followed by ischemic stroke. Tissue was collected $24 \mathrm{~h}$ after surgery (B) Infarct volume was quantitated by measuring absorbance of solvent extracted dye ${ }^{*} p<0.05$, one-way ANOVA). (C) Neuroscore at 0.5 and $24 \mathrm{~h}$ after stroke induction in mice pretreated with siRNA, MTfp-siRNA or PBS control. ${ }^{*} p<0.05$, ${ }^{* *} p<$ 0.001. Bottom Panel (D-F): Treatment with MTfp-scrambled RNA does not offer neuroprotection after ischemic stroke. (D) TTC-stained brain sections of mice receiving various IV injections, followed by ischemic stroke. (E) Infarct volume was quantitated by measuring absorbance $(485 \mathrm{~nm})$ of solvent extracted dye. Data are shown as mean \pm SEM (no significant difference). (F) Neuroscore at 0.5 and $24 \mathrm{~h}$ after stroke induction. Data are shown as mean \pm SEM (There is no significant difference between the treatments at either time point) ( $n=3$ mice per group).

examined by 3D confocal fluorescence microscopy (Figure 2) to determine whether MTfp could enable increased delivery of the siRNA across the BBB (i.e., the vasculature) to the brain parenchymal tissues (Figures 2A-C). We found the conjugation of MTfp to siRNA allowed increased localization of siRNA into the brain parenchyma compared to siRNA alone (Figure $2 \mathrm{~A}$ vs. $2 \mathrm{D}$ and Supplementary Table S1). Interestingly, a small amount of siRNA was able to cross the BBB (Figure 2F).

To demonstrate the therapeutic efficacy of cargo delivery to the CNS by MTfp, MTfp conjugated to NOX4-specific siRNA was examined in mouse model of ischemic stroke. Mice were dosed three times, at $1 \mathrm{~h}$ intervals, and then subjected to ischemic stroke in the left hemisphere for a duration of $1 \mathrm{~h}$ by middle cerebral artery occlusion (MCAO). In this proof-of-concept study, we chose to administer treatment (MTfp-siRNA, MTfp-scramRNA, NOX4 siRNA alone, and PBS controls) prior to stroke induction to demonstrate delivery across the intact $\mathrm{BBB}$ since the integrity will certainly be compromised by the invasive nature of stroke induction. Furthermore, this therapy is intended to reduce NOX4/hypoxia associated pathology and will have no effect if blood flow to the site of injury is impaired (i.e., blood clots or filament occlusion). Animals were sacrificed at $24 \mathrm{~h}$ post reperfusion. It is known that induction of ischemic stroke leads to elevated CNS expression of NOX4 (Kleinschnitz et al., 2010). This trend is seen in experiments, conducted by a different operators, in animals induced with stroke after being treated with PBS, NOX4 siRNA alone (Figure 3 and Supplementary Table S3) or MTfp-scramRNA control (Supplementary Tables S1, S2, S4). However, when treated with MTfp-siRNA, NOX4 upregulation was reduced after stroke, where NOX4 mRNA was only $80 \%$ of the levels observed with the PBS stroke-induced control (Figure 3).

Finally, we assessed whether MTfp-siRNA mediated NOX4 knockdown resulted in protection against neurological deterioration. Mice were treated and subjected to ischemic stroke as described above. Neuroscore (Jiang et al., 2005) was measured $0.5 \mathrm{~h}$ after removal of anesthesia (to determine surgical efficiency) and again at $24 \mathrm{~h}$ post stroke (to measure recovery and outcome). After the second behavioural assessment, animals were sacrificed and infarct volume was quantitated by 2,3,5-triphenyltetrazolium chloride (TTC) staining. Total infarct volume as well as motor deficits were measured (Figure 4; Supplementary Table S2). Compared to PBS controls, sham surgery, siRNA alone and MTfp-scramRNA controls, the MTfp-siRNA resulted in significantly smaller infarcts (Figure 4) and corresponded to less severe paralysis immediately after cessation of MCAO (Figure 4). Twenty-four hours after surgery, mice that had been treated with MTfpsiRNA achieved neurological scores of $<1$ which is functionally near normal, while the control animals remained mentally and physically impaired.

\section{DISCUSSION}

We chose to focus on stroke because it is a leading cause of death worldwide, and is a difficult disease to treat. The gene, NOX4, has been shown to be upregulated during stroke and thus NOX4 offers a convenient target to test the therapeutic effects of a MTfpdelivered siRNA. Currently, recombinant tissue plasminogen activator is the only approved drug for treatment of stroke but due to contraindications, it can only be used in about $10 \%$ of patients. Furthermore, mainstream approaches are turning to pretreament approaches that stabilize and reduce the brain damage in patients with recurrent stroke (Schmülling et al., 2003; Mackman, 2008; Fuentes et al., 2009; Milionis et al., 2009; Yoo, 2016; Tsivgoulis et al., 2018) or patients at a risk for stroke (Aoki and Uchino, 2011). Thus, approaches that limit stroke-related damage are emerging as important therapeutic tools. 
New and effective treatments are needed for reducing the frequency and severity of recurrent stroke. Oxidative stress, which is the damage caused by reactive oxygen species, appears to play a central role in neuronal death in stroke. NOX4 is one of the major sources of oxidative stress and therefore an excellent therapeutic target in acute stroke. Upon ischemia, NOX4 is highly induced in both human and mouse brains. In addition, following cerebral ischemia, mice genetically deficient in NOX4 $\left(\mathrm{Nox}_{4}^{-1-}\right.$ ) are largely protected from oxidative stress, neuronal death and BBB leakage (Kleinschnitz et al., 2010). Furthermore, NOX4 is a culprit in CNS disease besides stroke and therefore this POC may have utility in other brain pathologies (Kim et al., 2016). However, targeting NOX4 in the brain is challenging due to the difficulty in getting a therapeutic across the BBB. Therefore, we used the small peptide, MTfp, as a braintargeting carrier for NOX4 siRNA.

Our data, obtained using a murine stroke model with the POC targeted against NOX4 for the amelioration of stroke is an example of efficacious gene knockdown in a model of a disease resident in the brain in which the $\mathrm{BBB}$ is intact. In this proof-of-concept study we opted to deliver the treatment before stroke induction to assess this approach in reducing the severity of stroke. This was also done to show that MTfp can cross an intact BBB, since stroke, by definition, results in a disruption of the BBB. Using a novel stable isotopic liquid chromatography mass spectrometry methodology to measure the transport of MTfp into the brain, we have reported elsewhere the initial rate of entry as $2.1 \mathrm{nM} / \mathrm{min}$ (Eyford et al., 2019). Additionally, stroke impairs blood flow and it is possible that if therapies were to be administered IV during or after a stroke, they would be less likely to gain access to the affected tissues. We suspect that, to achieve best outcomes, the POC conjugate described here will have to be used in combination with traditional fibrolytic therapies. Despite the clinical limitations, the POC used in this study demonstrates that MTfp has the capability of granting access to the CNS, to a molecule (RNA) that would normally be excluded.

Previous attempts to deliver siRNA to the CNS have relied on antibody-targeted liposomes (Zhang et al., 2004) or non-covalent, electrostatic interactions with a peptide derived from rabies virus glycoprotein (Kumar et al., 2007; Kim et al., 2010; Alvarez-Erviti et al., 2011) or by intracerebroventricular injection into the brain (Helmschrodt et al., 2017). Free siRNA, and even many lipidencapsulated formulations, are quickly eliminated from the circulatory system to accumulate within organs, such as kidney and liver, and degraded by the mononuclear phagocyte system (Park et al., 2016). Furthermore, nanotechnology-based siRNA carriers such as protease-responsive, brain targeting nanoparticles (Guo et al., 2018) and others approaches (Mann et al., 2016; Tian et al., 2018), simply localise to the area of tissue damage and do not transcytosis across the $\mathrm{BBB}$ and thus have limited biodistribution. Thus they never reach the CNS in significant amounts to have a biological outcome, and this may lead to poor efficacy in a clinical setting. Some examples exist for the entry of free siRNA (Xie et al., 2020) or siRNAencapsulated nanoparticles ( $\mathrm{Li}$ et al., 2021) to enter the CNS, however, to date these intriguing approaches are only described to exert their therapeutic effect after a prior breach of the $\mathrm{BBB}$ and do not directly show transcytosis of the intact BBB. For example, some studies on traumatic brain injury indicate that BBB may be breached for several days after induction of trauma (Li et al., 2021), others indicate the BBB is permeable for weeks after initial injury (e.g., ischemia, blunt force trauma) (Strbian et al., 2008; Price et al., 2016), and some BBB tight junction components can be downregulated for up to 8 weeks (Nag et al., 2007). In addition, observation of brain penetration in Alzheimer's disease and Multiple Sclerosis models have shown a prior breach in the $\mathrm{BBB}$ as part of the pathogenesis of the disease (Ujiie et al., 2003; Bennett et al., 2010; Biron et al., 2011; Biron et al., 2013), and siRNA studies in these models similarly cannot be used to conclude transcytosis of siRNA across an intact BBB (Zhou et al., 2020). This is the scientific background that provided the premise for the current study. In this study the BBB is intact at the time of injection of the "nanomule" siRNA conjugate into the peripheral circulatory system and accumulates in significant enough quantities to ameliorate the pathogenic features of ischemic stroke after it is subsequently induced. In contrast, the POC we describe, MTFp, is connected covalently and can be produced using standard peptide/RNA synthesis and conjugation technologies. We have shown in another study that MTfp accumulates within the brain paranchyma at a rate of $2.1 \mathrm{nM} / \mathrm{min}$ after delivery, and that after only $30 \mathrm{~min}$, the accumulation of MTfp within the brain was at a ratio of $1 / 4$ of that found in the kidneys, which is extremely efficient for drug delivery across the BBB (Eyford et al., 2019). The MTfp is derived from a protein of human origin, further reducing the likelihood of immunogenicity compared to a viral carrier. It traverses the intact $\mathrm{BBB}$, transports without degradation, to be widely distributed in microglia, neurons and astroglia in the CNS, and is delivered in sufficient quantities to knockdown NOX4 and alleviate ischemic stroke. This approach represents a less invasive method for delivery of siRNA and other drug conjugates to the CNS and could conceivably be used as a platform to modify a number of diseases in the brain.

In conclusion, we describe the modulation of neuroinflammation in the central nervous system using an siRNA directed at NOX4 expressed in the resident brain cells. This discovery will be useful for understanding the mechanism leading to neuroinflammatory diseases such as stroke, and perhaps lead to pretreatment and post-stroke regimens. In addition, this work identifies a novel, non-invasive general method of ferrying therapeutic cargo across the $\mathrm{BBB}$ to dampen the oxidative stress triggering neuroinflammation that leads to many neuropathologies. Future focus will be on expanding the utility of this new carrier to traverse therapeutics across the battlement of the $\mathrm{BBB}$ for the treatment of other pathologies within the physiological fortress of the CNS.

\section{DATA AVAILABILITY STATEMENT}

The original contributions presented in the study are included in the article/Supplemental Material, further inquiries can be directed to the corresponding authors. 


\section{ETHICS STATEMENT}

The animal study was reviewed and approved by the Animal Care Committees of the National Research Council of Canada and the University of British Columbia; both of which are governed by the Canadian Council of Animal Care.

\section{AUTHOR CONTRIBUTIONS}

All authors contributed to the paper. BE and CS are co-first authors, and with WJ wrote the manuscript; all authors revised it and approved the final version. WJ conceived and supervised the project.

\section{FUNDING}

Funding for this work was provided to WJ in the Michael Smith Laboratories, at the University of British Columbia and the Vancouver Prostate Centre, at Vancouver General Hospital, by a grant from the Canadian Institutes for Health Research (MOP133635), a grant from the Natural Sciences and Engineering Research Council of Canada (CRDPJ 452456-13) in collaboration with Bioasis Technologies Inc. (BTI, meimei@ bioasis.us), a grant from the W. Garfield Weston Foundation (RR161038), and donations from the Sullivan Urology Foundation at Vancouver General Hospital. TA was supported by NIH grants 1S10OD010756-01A1 and

\section{REFERENCES}

Alvarez-Erviti, L., Seow, Y., Yin, H., Betts, C., Lakhal, S., and Wood, M. J. (2011). Delivery of siRNA to the mouse brain by systemic injection of targeted exosomes. Nat. Biotechnol. 29 (4), 341-345. doi:10.1038/nbt.1807

Aoki, J., and Uchino, K. (2011). Treatment of risk factors to prevent stroke. Neurotherapeutics 8 (3), 463-474. doi:10.1007/s13311-011-0054-0

Bennett, D. A., Krishnamurthi, R. V., Barker-Collo, S., Forouzanfar, M. H., Naghavi, M., Connor, M., et al. (2014). The global burden of ischemic stroke: findings of the GBD 2010 study. Glob. Heart 9 (1), 107-112. doi:10. 1016/j.gheart.2014.01.001

Bennett, J., Basivireddy, J., Kollar, A., Biron, K. E., Reickmann, P., Jefferies, W. A., et al. (2010). Blood-brain barrier disruption and enhanced vascular permeability in the multiple sclerosis model EAE. J. Neuroimmunol. 229 (12), 180-191. doi:10.1016/j.jneuroim.2010.08.011

Biron, K. E., Dickstein, D. L., Gopaul, R., Fenninger, F., and Jefferies, W. A. (2013). Cessation of neoangiogenesis in Alzheimer's disease follows amyloid-beta immunization. Sci. Rep. 3, 1354. doi:10.1038/srep01354

Biron, K. E., Dickstein, D. L., Gopaul, R., and Jefferies, W. A. (2011). Amyloid triggers extensive cerebral angiogenesis causing blood brain barrier permeability and hypervascularity in Alzheimer's disease. PLoS One 6 (8), e23789. doi:10.1371/journal.pone.0023789

Braasch, D. A., Paroo, Z., Constantinescu, A., Ren, G., Oz, O. K., Mason, R. P., et al. (2004). Biodistribution of phosphodiester and phosphorothioate siRNA. Bioorg. Med. Chem. Lett. 14 (5), 1139-1143. doi:10.1016/j.bmcl. 2003.12.074

Chiurchiù, V., Orlacchio, A., and Maccarrone, M. (2016). Is modulation of oxidative stress an answer? The state of the art of redox therapeutic actions in neurodegenerative diseases. Oxid. Med. Cell Longev. 2016, 7909380. doi:10. $1155 / 2016 / 7909380$
1S10OD018124-01A1. BE was supported by Postdoctoral Fellowships from the Michael Smith Foundation for Health Research, the Centre for Blood Research at the University of British Columbia and the Pacific Alzheimer's Foundation. CS was supported by an Alzheimer's Drug Discovery Foundation Outstanding Young Investigator Scholarship, and by a William and Dorothy Gilbert Graduate Scholarship in Biomedical Sciences at the University of British Columbia and a scholarship from the Centre for Blood Research Graduate Student Award at the University of British Columbia.

\section{ACKNOWLEDGMENTS}

We thank Terry Pearson from the University of Victoria, Department of Biochemistry for his comments on the manuscript; and Wade Edris for his technical assistance with confocal and deconvolution microscopy imaging. We also thank the National Research Council of Canada and Ava $\mathrm{McHugh}$ for their professional services. This manuscript was originally part of a study that has been released as a pre-print at bioRxiv (Eyford et al., 2019).

\section{SUPPLEMENTARY MATERIAL}

The Supplementary Material for this article can be found online at: https://www.frontiersin.org/articles/10.3389/fmolb.2021.611367/ full\#supplementary-material.

Cooney, S. J., Bermudez-Sabogal, S. L., and Byrnes, K. R. (2013). Cellular and temporal expression of NADPH oxidase (NOX) isotypes after brain injury. J. Neuroinflam. 10, 155. doi:10.1186/1742-2094-10-155

Demeule, M., Poirier, J., Jodoin, J., Bertrand, Y., Desrosiers, R. R., Dagenais, C., et al. (2002). High transcytosis of melanotransferrin (P97) across the bloodbrain barrier. J. Neurochem. 83 (4), 924-933. doi:10.1046/j.1471-4159.2002. 01201.x

Eyford, B. A., Singh, C. S. B., Abraham, T., Munro, L., Choi, K. B., Hildebrandt, R., et al. (2019). A nanomule peptide-siRNA conjugate that traverses the intact blood brain barrier and attenuates stroke. BioRxiv, 871186

Food, M. R., Rothenberger, S., Gabathuler, R., Haidl, I. D., Reid, G., and Jefferies, W. A. (1994). Transport and expression in human melanomas of a transferrinlike glycosylphosphatidylinositol-anchored protein. J. Biol. Chem. 269 (4), 3034-3040. doi:10.1016/s0021-9258(17)42043-6

Fuentes, B., Martínez-Sánchez, P., and Díez-Tejedor, E. (2009). Lipidlowering drugs in ischemic stroke prevention and their influence on acute stroke outcome. Cerebrovasc. Dis. 27 (Suppl. 1), 126-133. doi:10. $1159 / 000200450$

Global Burden of Disease Cancer CollabrationFitzmaurice, C., Allen, C., Barber, R. M., Barregard, L., Bhutta, Z. A., et al. (2017). Global, regional, and national cancer incidence, mortality, years of life lost, years lived with disability, and disability-adjusted life-years for 32 cancer groups, 1990-2015: a systematic analysis for the global burden of disease study. JAMA Oncol. 3 (4), 524-548. doi:10.1001/jamaoncol.2016. 5688

Guo, C., Sun, L., Chen, X., and Zhang, D. (2013). Oxidative stress, mitochondrial damage and neurodegenerative diseases. Neural Regen. Res. 8 (21), 2003-2014. doi:10.3969/j.issn.1673-5374.2013.21.009

Guo, X., Deng, G., Liu, J., Zou, P., Du, F., Liu, F., et al. (2018). Thrombinresponsive, brain-targeting nanoparticles for improved stroke therapy. ACS Nano 12 (8), 8723-8732. doi:10.1021/acsnano.8b04787 
Helmschrodt, C., Höbel, S., Schöniger, S., Bauer, A., Bonicelli, J., Gringmuth, M., et al. (2017). Polyethylenimine nanoparticle-mediated siRNA delivery to reduce a-synuclein expression in a model of Parkinson's disease. Mol. Ther. Nucleic Acids 9, 57-68. doi:10.1016/j.omtn.2017.08.013

Huggett, J., Dheda, K., Bustin, S., and Zumla, A. (2005). Real-time RT-PCR normalisation; strategies and considerations. Genes Immun. 6 (4), 279-284. doi:10.1038/sj.gene.6364190

Jiang, S. X., Lertvorachon, J., Hou, S. T., Konishi, Y., Webster, J., Mealing, G., et al. (2005). Chlortetracycline and demeclocycline inhibit calpains and protect mouse neurons against glutamate toxicity and cerebral ischemia. J. Biol. Chem. 280 (40), 33811-33818. doi:10.1074/jbc.M503113200

Karkan, D., Pfeifer, C., Vitalis, T. Z., Arthur, G., Ujiie, M., Chen, Q., et al. (2008). A unique carrier for delivery of therapeutic compounds beyond the blood-brain barrier. PLoS One 3 (6), e2469. doi:10.1371/journal.pone.0002469

Kim, J., Seo, M., Kim, S. K., and Bae, Y. S. (2016). Flagellin-induced NADPH oxidase 4 activation is involved in atherosclerosis. Sci. Rep. 6, 25437. doi:10. 1038/srep25437

Kim, S. S., Ye, C., Kumar, P., Chiu, I., Subramanya, S., Wu, H., et al. (2010). Targeted delivery of siRNA to macrophages for anti-inflammatory treatment. Mol. Ther. 18 (5), 993-1001. doi:10.1038/mt.2010.27

Kleinschnitz, C., Grund, H., Wingler, K., Armitage, M. E., Jones, E., Mittal, M., et al. (2010). Post-stroke inhibition of induced NADPH oxidase type 4 prevents oxidative stress and neurodegeneration. PLoS Biol. 8 (9), e1000479. doi:10.1371/ journal.pbio.1000479

Kowal, S. L., Dall, T. M., Chakrabarti, R., Storm, M. V., and Jain, A. (2013). The current and projected economic burden of Parkinson's disease in the United States. Mov. Disord. 28 (3), 311-318. doi:10.1002/mds.25292

Kumar, P., Wu, H., McBride, J. L., Jung, K. E., Kim, M. H., Davidson, B. L., et al. (2007). Transvascular delivery of small interfering RNA to the central nervous system. Nature 448 (7149), 39-43. doi:10.1038/nature05901

Li, B., Bedard, K., Sorce, S., Hinz, B., Dubois-Dauphin, M., and Krause, K. H. (2009). NOX4 expression in human microglia leads to constitutive generation of reactive oxygen species and to constitutive IL-6 expression. J. Innate Immun. 1 (6), 570-581. doi:10.1159/000235563

Li, W., Qiu, J., Li, X. L., Aday, S., Zhang, J., Conley, G., et al. (2021). BBB pathophysiology-independent delivery of siRNA in traumatic brain injury. Sci. Adv. 7 (1), eabd6889. doi:10.1126/sciadv.abd6889

Ma, M. W., Wang, J., Zhang, Q., Wang, R., Dhandapani, K. M., Vadlamudi, R. K., et al. (2017). NADPH oxidase in brain injury and neurodegenerative disorders. Mol. Neurodegener. 12 (1), 7. doi:10.1186/s13024-017-0150-7

Mackman, N. (2008). Triggers, targets and treatments for thrombosis. Nature 451 (7181), 914-918. doi:10.1038/nature06797

Mann, A. P., Scodeller, P., Hussain, S., Joo, J., Kwon, E., Braun, G. B., et al. (2016). A peptide for targeted, systemic delivery of imaging and therapeutic compounds into acute brain injuries. Nat. Commun. 7, 11980. doi:10. 1038/ncomms11980

Milionis, H. J., Giannopoulos, S., Kosmidou, M., Panoulas, V., Manios, E., Kyritsis, A. P., et al. (2009). Statin therapy after first stroke reduces 10 year stroke recurrence and improves survival. Neurology 72 (21), 1816-1822. doi:10.1212/ WNL.0b013e3181a711cb

Moroo, I., Ujiie, M., Walker, B. L., Tiong, J. W., Vitalis, T. Z., Karkan, D., et al. (2003). Identification of a novel route of iron transcytosis across the mammalian blood-brain barrier. Microcirculation 10, 457-462. doi:10.1038/ sj.mn.7800213

Murray, C. J., Atkinson, C., Bhalla, K., Birbeck, G., Burstein, R., Chou, D., et al. (2013). The state of United States health, 1990-2010: burden of diseases, injuries, and risk factors. JAMA 310 (6), 591-608. doi:10.1001/jama.2013. 13805

Nag, S., Venugopalan, R., and Stewart, D. J. (2007). Increased caveolin-1 expression precedes decreased expression of occludin and claudin- 5 during blood-brain barrier breakdown. Acta Neuropathol. 114 (5), 459-469. doi:10.1007/s00401007-0274-x

Park, J., Park, J., Pei, Y., Xu, J., and Yeo, Y. (2016). Pharmacokinetics and biodistribution of recently-developed siRNA nanomedicines. Adv. Drug Deliv. Rev. 104, 93-109. doi:10.1016/j.addr.2015.12.004

Price, L., Wilson, C., and Grant, G. (2016). Chapter 4 blood-brain barrier pathophysiology following traumatic brain injury. Boca Raton, FL: CRC Press/Taylor and Francis Group, 299.
Qin, Y. Y., Li, M., Feng, X., Wang, J., Cao, L., Shen, X. K., et al. (2017). Combined NADPH and the NOX inhibitor apocynin provides greater anti-inflammatory and neuroprotective effects in a mouse model of stroke. Free Radic. Biol. Med. 104, 333-345. doi:10.1016/j.freeradbiomed.2017.01.034

Radermacher, K. A., Wingler, K., Langhauser, F., Altenhöfer, S., Kleikers, P., Hermans, J. J., et al. (2013). Neuroprotection after stroke by targeting NOX4 as a source of oxidative stress. Antioxid. Redox Signal. 18 (12), 1418-1427. doi:10. 1089/ars.2012.4797

Rios, A. M. (2019). Mexico: most common cause of death 2017. Available at: https://www.statista.com/statistics/960030/mexico-causes-death/ (Accessed December 10, 2019)

Rothenberger, S., Food, M. R., Gabathuler, R., Kennard, M. L., Yamada, T., Yasuhara, O., et al. (1996). Coincident expression and distribution of melanotransferrin and transferrin receptor in human brain capillary endothelium. Brain Res. 712 (1), 117-121. doi:10.1016/0006-8993(96) $88505-2$

Rubiano, A. M., Carney, N., Chesnut, R., and Puyana, J. C. (2015). Global neurotrauma research challenges and opportunities. Nature 527 (7578), S193-S197. doi:10.1038/nature16035

Schmülling, S., Rudolf, J., Strotmann-Tack, T., Grond, M., Schneweis, S., Sobesky, J., et al. (2003). Acetylsalicylic acid pretreatment, concomitant heparin therapy and the risk of early intracranial hemorrhage following systemic thrombolysis for acute ischemic stroke. Cerebrovasc. Dis. 16 (3), 183-190. doi:10.1159/ 000071114

Statistics Canada (2018). Leading causes of death total population, by age group 2018. National center for health statistics. Available at: https://www150. statcan.gc.ca/t1/tbl1/en/tv.action?pid=1310039401 (Accessed July 26, 2018).

Strbian, D., Durukan, A., Pitkonen, M., Marinkovic, I., Tatlisumak, E., Pedrono, E., et al. (2008). The blood-brain barrier is continuously open for several weeks following transient focal cerebral ischemia. Neuroscience 153 (1), 175-181. doi:10.1016/j.neuroscience.2008.02.012

Thom, G., Tian, M. M., Hatcher, J. P., Rodrigo, N., Burrell, M., Gurrell, I., et al. (2019). A peptide derived from melanotransferrin delivers a protein-based interleukin 1 receptor antagonist across the $\mathrm{BBB}$ and ameliorates neuropathic pain in a preclinical model. J. Cereb. Blood Flow Metab. 39 (10), 2074-2088. doi:10.1177/0271678X18772998

Tian, T., Zhang, H. X., He, C. P., Fan, S., Zhu, Y. L., Qi, C., et al. (2018). Surface functionalized exosomes as targeted drug delivery vehicles for cerebral ischemia therapy. Biomaterials 150, 137-149. doi:10.1016/j.biomaterials. 2017.10.012

Tsivgoulis, G., Goyal, N., Kerro, A., Katsanos, A. H., Krishnan, R., Malhotra, K., et al. (2018). Dual antiplatelet therapy pretreatment in IV thrombolysis for acute ischemic stroke. Neurology 91 (11), e1067-e76. doi:10.1212/WNL. 0000000000006168

Ujiie, M., Dickstein, D. L., Carlow, D. A., and Jefferies, W. A. (2003). Bloodbrain barrier permeability precedes senile plaque formation in an Alzheimer disease model. Microcirculation 10, 463-470. doi:10.1038/sj. $\mathrm{mn} .7800212$

Whiteford, H. A., Ferrari, A. J., Degenhardt, L., Feigin, V., and Vos, T. (2015). The global burden of mental, neurological and substance use disorders: an analysis from the global burden of disease study 2010. PLoS One 10 (2), e0116820. doi:10.1371/journal.pone.0116820

Xie, J., Hong, E., Ding, B., Jiang, W., Zheng, S., Xie, Z., et al. (2020). Inhibition of NOX4/ROS suppresses neuronal and blood-brain barrier injury by attenuating oxidative stress after intracerebral hemorrhage. Front. Cell Neurosci. 14, 578060. doi:10.3389/fncel.2020.578060

Xu, J., Murphy, S. L., Kochanek, K. D., Bastian, B. A., and Deaths, A. E. (2018). Deaths: final data for 2013. Natl. Vital Stat. Rep. 64 (5), 1-119. PMID: 30248015

Yang, J., Tiong, J., Kennard, M., and Jefferies, W. A. (2004). Deletion of the GPI pre-anchor sequence in human $p 97-$ a general approach for generating the soluble form of GPI-linked proteins. Protein Expr. Purif. 34 (1), 28-48. doi:10. 1016/j.pep.2003.09.007

Yao, H., Ago, T., Kitazono, T., and Nabika, T. (2017). NADPH oxidase-related pathophysiology in experimental models of stroke. Int. J. Mol. Sci. 18 (10), 2123. doi:10.3390/ijms18102123

Yoo, A. J. (2016). Imaging-based patient selection for intra-arterial stroke therapy. Amsterdam, Netherlands: Universiteit vanAmsterdam, 5, 84-96. 
Zhang, Y., Zhang, Y. F., Bryant, J., Charles, A., Boado, R. J., and Pardridge, W. M. (2004). Intravenous RNA interference gene therapy targeting the human epidermal growth factor receptor prolongs survival in intracranial brain cancer. Clin. Cancer Res. 10 (11), 3667-3677. doi:10.1158/1078-0432.CCR03-0740

Zhou, Y., Zhu, F., Liu, Y., Zheng, M., Wang, Y., Zhang, D., et al. (2020). Blood-brain barrier-penetrating siRNA nanomedicine for Alzheimer's disease therapy. Sci. Adv. 6 (41). doi:10.1126/sciadv.abc7031

Conflict of Interest: The authors declare that this study received funding from Bioasis Technologies Inc. The funder did not influence the study design, collection, analysis, interpretation of data, the writing of this article or the decision to submit it for publication.

Copyright $(2021$ Eyford, Singh, Abraham, Munro, Choi, Hill, Hildebrandt, Welch, Vitalis, Gabathuler, Gordon, Adomat, Guns, Lu, Pfeifer, Tian and Jefferies. This is an open-access article distributed under the terms of the Creative Commons Attribution License (CC BY). The use, distribution or reproduction in other forums is permitted, provided the original author(s) and the copyright owner(s) are credited and that the original publication in this journal is cited, in accordance with accepted academic practice. No use, distribution or reproduction is permitted which does not comply with these terms. 